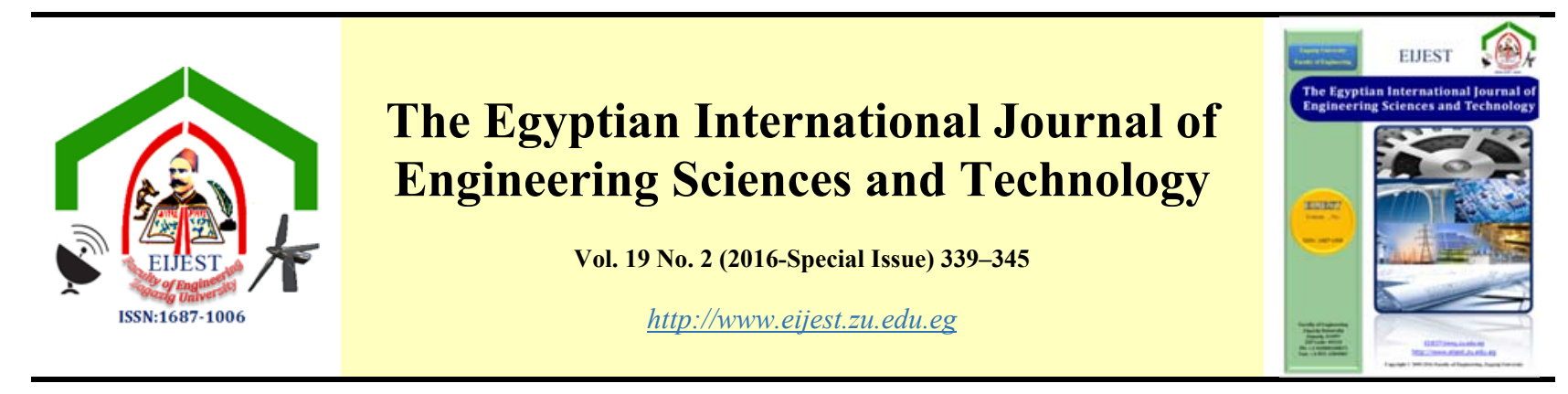

\title{
Analytical Approach for Routing Guidance System Considering Vehicular Emissions
}

\author{
Ahmed Elkafoury ${ }^{\mathrm{a}}$ and Abdelazem Negm ${ }^{\mathrm{b}}$ * \\ ${ }^{a}$ Faculty of Engineering-Tanta University, Elgesh st.,Tanta, Egypt. E-MAIL: kaforiengin@inbox.com \\ ${ }^{b}$ Egypt-Japan University of Science and Technology (E-JUST), New Borg Elarab City-Alexandria, Egypt, PostalCode 21934
}

\begin{tabular}{l}
\hline A R T I C L E I N F O \\
\hline Article history: \\
Received 28 May 2016 \\
Accepted 27 June 2016 \\
Available online 1 July 2016 \\
\hline
\end{tabular}

Keywords:

Traffic control

Eco-routing

Travel costs

Fuzzy optimization

\begin{abstract}
A B S T R A C T
Involving the environmental dimension in traffic control methods is the recent trend in transportation research. This paper introduces an eco-routing method that searches for route optima to target the social optimality but considering the user preference as well. A negotiate trade-off between four travel-costs is considered for the route optimality. The four travel costs are the vehicular emission cost, the user instinctive, traffic performance, and the user preference. Fuzzy multi-objective decision optimization is utilized for the decision model to select the optimal route from the fuzzy set of available alternatives between the origin and the destination. The introduced method outperforms the literature eco-routing methods that overemphasizes the most weighted criteria and neglects the decrease of marginal utility or consider the environmental dimension only as a constrain for the travel time minimization problem.
\end{abstract}

\section{Introduction}

Route for his or her to start a trip or to adjust the route of an already started trip. They are sub-systems of Driver Assistance Systems (DAS) which is a component of Intelligent Transportation Systems (ITS)[1]. It aims to improve the travel time, traffic safety, mobility, environmental pollution, or customer satisfaction. In route guidance, real time collection of vehicle information and traffic state indicators is performed using and advanced information systems in addition to communication technologies. This information is sent to the routing algorithm to recommend the optimal path to achieve great performance for the network as a whole, or a user optimum where no road user can change its own route to a faster route[2]-[4]. In this concern, the optimization framework of the route guidance can be classified to:

Network system optimal route guidance: this system can be described as a management system more than a route guidance system. In which the entire network is the physical decision consideration. It tends to improve the travel situation over the entire network. Therefore, not always the user's optimal solution is the decision of the route guidance system. In other words, the user' may be guided to take a sub-optimal route in order to improve the performance of the network. Therefore, the drivers in this system have complains as their cost is not optimized.

User optimum route guidance: this system is

\footnotetext{
* Corresponding author. Tel.: +2-010-573-5345; fax: +203-459-9520.

E-mail address: negm@ejust.edu.eg, amnegm@zu.edu.eg.
} 
oriented for the user's satisfaction. It optimizes the individual optimal paths considering the driver's marginal cost in terms of travel distance or travel time without considering the optimality of the entire network. i.e., the total cost of society not minimized. And in most cases, it's oriented to users who are equipped to receive. This route guidance system,

Pollution related to traffic operations can be reduced if vehicles follow environmentally friendly paths. Since it is proved that it is not always the time or distance shortest path is always minimize fuel consumption [5], [6], the eco-routing problem is more dimensioned than the ordinary time or distance minimization problem. In regard,( E. Ericsson et al., 2006 )[7]developed an eco-routing model based on standard shortest path algorithm is used to calculate the optimal path that minimizes the environmental impacts only. (K. Boriboonsomsin and M. Barth, 2012) [8] considered also the emission as the decision variable to estimate the optimal shortest path that minimized emissions based on historical trajectory data and real-time data from wireless sensors, probe vehicles, and loop detectors. Both of them did not consider the effect of flow interruption due to traffic signals. (H. Rakha et al., 2012) [9] introduced a simulation based framework for eco-routing to minimize vehicle emissions based on simulation approach. In this framework, only the cursing speed is considered for emission estimations neglecting the effect of acceleration and deceleration.( A. Chen et al., 2011)[10] considered the $\mathrm{CO}$ emission as a constrain for the traffic assignment model routing problem without a general formula for different pollutant types. (Y. Nie and Q. Li)[11] minimized the total monetaryweighted travel cost of travel time, speed, fuel consumption, and emission. (J. Sun and H. X. Liu, 2015) [12]incorporated a microscopic vehicle emission model into a Markov Decision Process (MDP) in an eco-routing model recognizing the effect of the traffic signals on the vehicle trajectory. But, they considered only the environmental cost as the decision variable in the model.

The criticism of previous research works in ecorouting optimization problem lead to the following:

1. Most of developed methods use the emission as the individual decision variable that is minimized. This means searching for network optimum and not considering the user preference

2. Some eco-routing models consider different costs and deals with the emission as constrain for the system and not a decision variable.

3. Multi objective eco-routing models consider weighted average of different costs as the decision variable. This overemphasizes the criterion that is highly weighted neglecting the reduction in marginal utility for the system.

This paper proposes a descriptive optimization method that addresses the urban traffic eco-routing problem. It supposes a system to minimize the total cost but considering the users benefit. This method incorporates a fuzzy decision optimization for the decision model. The model search for optima for the eco-routing problem by a trade-off between four travel cost. The four travel costs are the environmental cost, the user instinctive, traffic performance, and the user preference. This fuzzy trade-off overcomes the disadvantage of optimizing multi-objective weighting models.

\section{Network definition}

Let $\mathrm{G}(\mathrm{N}, \mathrm{A})$ is the directed graph of the network with a set $\mathrm{N}$ of nodes and a set $\mathrm{A}$ of links. The problem is modeled as finding the optimal route from an origin node $s \in N$ to a destination node $d \in N$ where $s \neq d$. If there are $\mathrm{n}$ possible routes between $\mathrm{s}$ and $\mathrm{d}$, these routes are remarked as $r 1, r 2, r 3, \ldots, r n$. Each route $r \in R$ where $\mathrm{R}$ is the set of all acyclic routes from $\mathrm{s}$ to $\mathrm{d}$. Each $\mathrm{r}$ composes of successive arcs $\mathrm{a} \in \mathrm{A}$. These arcs are connected by nodes $c \in N$. Then, the router is represented as $\mathrm{r}$ (gr, a). If the number of arcs on the route $r$ are $m$, then the set of the arcs a is expressed as $\{a 1, a 2, a 3, \ldots, a m\}$. And the set gr $\subset$ Nis expressed as $\mathrm{gr}=\{\mathrm{s}, \mathrm{c} 1, \mathrm{c} 2, \mathrm{c} 3, \ldots, \mathrm{cm}-1, \mathrm{~d}\}$. In the eco routing problem, the optimal route is a rout $r^{*} \in R$.

In the routing problem in this paper, there are 4 travel cost on each $r \in R$. They are, the environmental cost, the user incentive, traffic performance, and the user preference. The first and third cost represents the social cost while the second and fourth are related to the user benefit. The following parts show the mathematical formulation and notations used in each of them.

\section{Emission cost}

In the eco-routing problem, the focal point is on the environmentally related cost of travel. Seeing that the routing decision is determined on individual base and with a short time frame, the emission calculation should be estimated on a meso-scale. In which, the emission calculations of any vehicle on an arc is projected using the speed and acceleration over different segments of the arc. i.e. the vehicle trajectory. 
The key element to an eco-routing problem in urban street network is estimating the vehicle trajectoriesIn urban traffic networks, the existence of traffic signals at intersection is a primary cause of change of vehicle trajectory. Assuming an intersection $I \in N$ connecting between two $\operatorname{arcs}(a \in A)$ and $(a+1 \in A)$. In order to capture the vehicle trajectory over a route $r \in R$, the driving statues of the routing vehicle will be estimated over different route segments. This estimation is based on some assumptions which are:

1-The vehicle acceleration and deceleration rates are constant.

2-The length of any arc a is sufficient to accelerate from zero speed to the arc average speed and also sufficient to decelerate from the average speed to zero speed.

3-When the vehicle reached at non-signalized intersection $\mathrm{I} \square \mathrm{N}$, the vehicle is assumed to pass the intersection with the average speed.

Let the driving status is noted as $Y z$. At the signalized intersection the vehicle may experience one of the following two driving status. The first is the average speed driving (Y1). This status exists when the vehicle reaches the intersection $I$ during the green phase. It will pass the intersection with the average speed of the arc $a$. The second is the idling driving status (Y2). If the vehicle reached during the red phase, it will decelerate on the arc $a$ to stop at the intersection $I$. In this case, on the following arc $(a+1)$, the vehicle will accelerate again to the average speed of the arc $(a+1)$ when the signal turns to the green light.

On the other hand, the interruption effect of the traffic signal on a vehicle on an arc may transfer it to one of the following driving statuses:

1. Decelerating $(Y 3)$ : this driving status exists on the arc if the vehicle is decelerating to stop at the intersection $I$ because of the red light. For convenience, the distance required to full deceleration to zero speed on any arc $a \in A$ is noted as $l_{Y 3}$

2. Accelerating (Y4): this happens if the vehicle is passing the intersection I after full idling at it. For convenience, the distance required to full deceleration to zero speed on any $\mathrm{a} \in \mathrm{A}$ is noted as $l_{Y 4}$.

The value of $l_{Y 3}$ and $l_{Y 4}$ can be determined using the following equations:

$$
\begin{aligned}
& l_{Y 3}=\frac{v_{a}^{2}}{2 g(k \pm G)} \\
& l_{Y 4}=\frac{v_{a}^{2}}{2 g(k \mp G)}
\end{aligned}
$$

As the value of $v_{a}, g, k, G$ are the arc average travel speed, acceleration of gravity, specific coefficient of friction between vehicles tires and the wearing surface of the road, and the road longitudinal gradient in decimal respectively. $(k+G)$ is used if the arc has an upward gradient in $l_{Y 3}$ formula and if the arc has a downward gradient in the $l_{Y 4}$ formula. On contrary, $(k$ - $G$ )is used if the arc has a downward gradient in the $l_{Y 3}$ formula and if the arc has an upward gradient in the $l_{Y 4}$ formula.

In fact, the average travel speed provision problem, especially on signalized arterials, is a challenging task because of the interrupted nature of urban traffic flows. It is not only dependent for the travel time, traffic flow and occupancy, but also ultimately affected by the signal timing and conflicting traffic from cross streets. With the help of traffic simulation, the average speed can be provisioned if the travel demand, vehicle occupancies, and the signal status are provided. To the best of our knowledge, positive achievements have been reached regarding this issue. The review illustrates that Bongsoo Son et al., (2005) [13] introduced average travel speed estimated by conventional technique for the signalized intersection networks.

In the routing model, the arriving time at the intersection is vital for the driving status estimation and consequently, the vehicle trajectory appraisal. The reason is that if the time arriving at the intersection coincides with a red light phase of the traffic signal, the vehicle will decelerate and then idle at the intersection and finally accelerate again. Overall, it may be said that the vehicle will examine three driving status at the intersection $(Y 2, Y 3$, and $Y 4)$ instead of one driving status $(Y 1)$ in case of reaching the intersection at time of green phase.

If it is denoted that the acceleration and deceleration rates are $X_{a c}$ and $X_{d s}$ respectively, the time needed for deceleration from $v_{a}$ to zero $\left(t_{Y 3}\right)$ and for acceleration from zero speed to $v_{a}\left(t_{Y 4}\right)$ on any arc $a$ is updated as:

$$
\begin{aligned}
& t_{Y 4}=\frac{v_{a}}{X_{a c}} \\
& t_{Y 3}=\frac{v_{a}}{X_{d c}}
\end{aligned}
$$


and next, the travel time over the rest of the $\operatorname{arc} a$ is obtained by discounting $l_{Y 3}$ and $l_{Y 4}$ from $t_{a}$. To determine the exact arriving time at any intersection, first, the vehicle is assumed to move without interruption from the starting node of the arc (node $c$ ) at time $T^{c}$ until it reaches the ending node of the arc $($ node $c+1)$ at time $T^{c+1}=\frac{l_{a}}{v_{a}}$.i.e., experiencing one driving status $(Y 1)$. Then, to determine the exact arriving time $\left(T_{*}^{c+1}\right)$, a correction value $\Delta_{1}$ and $\Delta_{2}$ are add to $T^{c+1}$ according to the intersection type at $c$ and $(c+1)$ (signalized or not signalized) and according to coincidence of the arrival time with the green time interval or the red time interval $\left[T_{o}, T_{o}+T_{\text {red }}\right]$ at the signalized intersection. Where $T_{o}$ is the start time of the red phase and $T_{\text {red }}$ is the signal red phase duration. The following equations update the values of $\Delta_{1}, \Delta_{2}$, and $T_{*}^{c+1}$ :

$\Delta_{1}=\left\{\begin{array}{c}\left(t_{Y 4}-\frac{l_{Y 4}}{v_{a}}+T_{\text {red }}\right) \\ \text { ifc is signalized intersection and } \\ T_{o} \leq T^{c+1} \leq T_{o}+T_{\text {red }} \\ 0 \quad \text { otherwise }\end{array}\right.$

$\Delta_{2}=\left\{\begin{array}{c}\left(t_{Y 3}-\frac{l_{Y 3}}{v_{a}}\right) \\ i f(c+1) \text { is signalized intersection and } \\ T_{o} \leq T^{c+1} \leq T_{o}+T_{\text {red }} \\ \text { otherwise }\end{array}\right.$

The exact arriving time $\left(T_{*}^{c+1}\right)$ is figured out by the formula:

$T_{*}^{c+1}=T^{c+1}+\Delta_{1}+\Delta_{2}$

Along with $\Delta_{1}$ the value of $\Delta_{2}$ the vehicle driving status is resolved. If $\Delta_{1}$ is zero, the vehicle will not examine an acceleration at the arc staring point $c$. i.e., it will pass the intersection at $c$ with the average speed of the arc. Otherwise, it will accelerate. And if $\Delta_{2}$ is zero, the vehicle will not decelerate at the arc end point $(c+1)$. i.e., it will pass the intersection at $(c+1)$ with the average speed of the arc. Otherwise, it will decelerate. Figure. 1. Illustrates an example of the vehicle trajectory on a signalized route connecting a start node $s$ and a destination $d$ based on the driving status of different arcs where $s \neq d$.

The approach of estimating vehicular emissions is based on the instantaneous emission model VT-Meso presented by Rakha et al., 2015 [14]. In which, modal model that estimates the vehicle fuel consumption and emission rates on a link-by-link basis has been proposed. The model assumptions are the same as for our assumptions regarding acceleration and decelerating with constant rates. And between acceleration and deceleration, VT-Meso assumes that a vehicle travels at a constant cruise speed which is noted in this $\mathrm{c}$ as the arc average speed $\left(v_{a}\right)$. In fact, the cursing speed is equal to the average speed of the arc only if there are no stops, but in this study it is assumed that the cursing speed is equal to the average speed as an approximation.

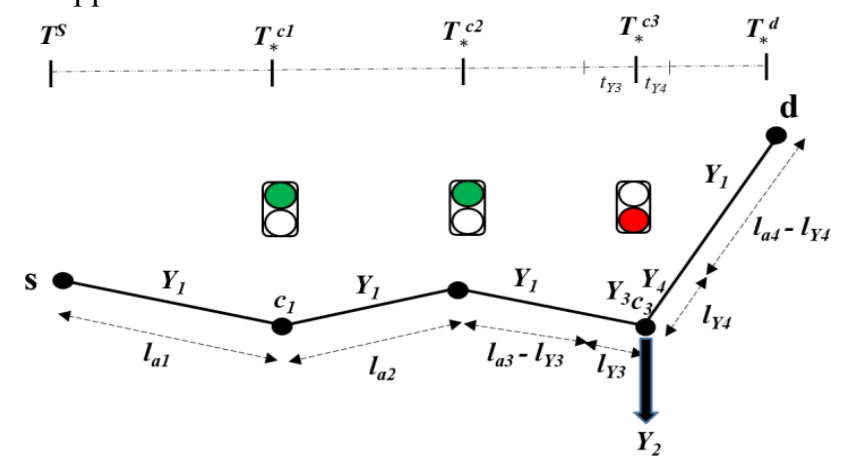

Figure 1: Example of vehicle trajectory on a route between two nodes

VT-Meso obtains the overall emission rate of a pollutant by integrating the instantaneous hot stabilized emission rates provided by the microscopic emission model VT-Micro [15] over the entire duration of a driving status. The hot stabilized emissions rates $\left(E F^{Y z}\right)$ of the pollutant $i\left(\mathrm{HC}, \mathrm{CO}, \mathrm{NO}_{\mathrm{x}}\right.$, or $\mathrm{CO}_{2}$ ) at any driving status $Y z$ are computed by applying VT-Meso for two types of vehicles; lightduty gasoline vehicles and trucks according to the following equations:

$$
\begin{aligned}
& E F_{i}^{Y 1}=\exp \left(k_{0,0}^{e}+k_{1,0}^{e} v_{a}+k_{2,0}^{e} v_{a}{ }^{2}+k_{3,0}^{e} v_{a}^{3}\right) \\
& E F_{i}^{Y 2}=\exp \left(k_{0,0}^{e}\right) \\
& E F_{i}^{Y 3}=\exp \left(d_{o}+d_{1} v_{a}+d_{2} v_{a}{ }^{2}+d_{3} v_{a}{ }^{3}+d_{4} v_{a}{ }^{4}+\right. \\
& \left.d_{5} v_{a}{ }^{5}+d_{6} v_{a}^{6}\right) \\
& E F_{i}^{Y 4}=\exp \left(a_{o}+a_{1} v_{a}+a_{2} v_{a}{ }^{2}+a_{3} v_{a}{ }^{3}+a_{4} v_{a}^{4}+\right. \\
& \left.a_{5} v_{a}{ }^{5}+a_{6} v_{a}{ }^{6}\right)
\end{aligned}
$$

Here, $E F_{i}^{Y 1}, E F_{i}^{Y 2}, E F_{i}^{Y 3}, E F_{i}^{Y 4}$ are the pollutant $i$ emission rate $(\mathrm{mg} / \mathrm{s})$ during average speed cursing, idling, decelerating, and accelerating respectively. $d_{0}, \ldots, d_{6}$ and $a_{0}, \ldots, a_{6}$, and $k_{0,0}^{e}, \ldots, k_{3,0}^{e}$ are vehiclespecific regression coefficients for the deceleration, acceleration, and cursing driving status respectively. The average speed of the arc $\left(v_{a}\right)$ in these equations is used in $(\mathrm{km} / \mathrm{h})$ units. In computing the entire emissions routing over a route $r$ based on the request of an origin $s$ and destination $d$ pair, the rates given by former equations are then multiplied by the total duration of 
the status. Then, over the route $r \in R$, the total emitted amount of pollutant $i\left(E_{i}(r)\right)(\mathrm{mg})$ is figured out as:

$$
\begin{aligned}
& E_{i}(r)=\left(\sum_{a \in r} \sum_{Y Z \in a} E F^{Y z}{ }_{i} t_{Y Z}\right)+ \\
& \sum_{\mathrm{c} \in g r}\left(E F^{Y 2}{ }_{i} t_{Y 2}\right) \quad \forall r \in R, \forall z \in\{1,3,4\}
\end{aligned}
$$

Finally, the emission optimality $E_{\text {iopt }}$ ration is the ration between $E_{i}(r)$ and the maximum allowable amount of pollutant during any trip $E_{i}(u l t)$ that is determined by specification and environmental laws in the area.

$$
E_{\text {iopt }}(r)=\frac{E_{i}(r)}{E_{i}(u l t)} \quad \forall r \in R
$$

\section{User incentive}

The user's decision for the routing problem is based on individual preferences while choosing the travel route. From the user's standpoint, the travel time is the solely crucial decision variable to determine a route for the trip. The user equilibrium mechanism does not care for the social benefit trough the emission reduction. Route with the shortest travel time is the outstanding for the user. In order to promote the users to shift to the eco-rout, other dimension should be incorporated to incentive the user. If the eco-rout is consuming less fuel, the user will go for it by virtue of saving money.

To form this incentive problem, a fuel consumption discount award system is used as a mean of encouraging. Initially, the fuel consumption rate in any driving status $\left(F C^{Y_{z}}\right)(L / s) Y z$ can be estimated using the VT-Meso model. It applies the same forms of the emission factor equations but uses the fuel consumption vehicle-specific regression coefficients for the deceleration, acceleration, and cursing driving status. Next, over the route $r \in R$, the total amount of fuel consumed by the routing vehicle Fuel(r) is computed using the following form:

$\operatorname{Fuel}(r)=\left(\sum_{a \in r} \sum_{Y Z \in a} F C^{Y z} t_{Y Z}\right)+$

$\sum_{\mathrm{c} \in g r}\left(F C^{Y 2} t_{Y 2}\right) \quad \forall r \in R, \quad \forall z \in\{1,3,4\}$

In light of this, a fuel consumption optimality indicator $\theta(r)$ is introduced to assess the quality of the economic incentive on the route $r . \theta(r)$ is calculated as:

$\theta(r)=\left(\frac{\text { Fuel }(r)}{\text { min Fuel }}\right) \quad \forall r \in R$

where minFuel is the fuel consumption on the optimal route from $s$ to $d$ when considering only the travel time as the decision variable. For example, if $\theta(r)=0.3$, then the economic cost of fuel consumed on the eco route $r$ is $70 \%$ less than that amount consumed if traveled on the optimal route from $s$ to $d$ when considering only the travel time as the decision variable.

\section{Traffic performance}

In order to consider the traffic performance criteria during the rout guidance decision, a traffic model is presented in this section. In which the focus is on the traffic density which is used as a convenient performance indicator of the stream. In this research, balancing the traffic densities on all available routes is approved to consider the traffic performance. This is in order to reduce the traffic congestions over the network. Handling the travel speed of the arc $a$, the traffic density $\rho_{a}$ can simply be updated using Greenshields model [16] shown in the following equation:

$v_{a}=v_{f}\left(1-\frac{\rho_{a}}{\rho_{a \max }}\right)$

where $v_{f}$ and $\rho_{a}$ max are the average speed and the jam density of the arc $a$. Next, the traffic load $(H(r))$ indicator is used as the traffic density factor. It can be estimated by measuring the ratio between the average of all traffic density of $a \in r$ and the average of all maximum traffic densities of $a \in r$. This is formed by the following equation:

$H(r)=\frac{\overline{\rho_{a}}}{\overline{\rho_{\text {amax }}}} \quad \forall a \in r$

Higher values of $H$ indicate worse traffic situation as the route $r$ tends to be saturated.

\section{User preference}

Given that a user will use the route $r$, the predicted time cost $\Phi(r)$ for traveling on this path is estimated using the calculated exact arriving time at the destination $d\left(T_{*}^{d}\right)$, and the routing start time at the origin node $s\left(T^{s}\right)$ as follow:

$\Phi(r)=T_{*}^{d}-T^{s} \forall \quad r \in R$

The routing model in this research tries to decrease the user dissatisfaction in terms of the travel time cost. The user is not satisfied with the travel time of the trip if it is longer than his or her anticipated travel time on the route. The dissatisfaction function can be expressed as follows: 
$F(r)=\left\{\begin{array}{lr}0 & \Phi \leq I \\ 1 & \Phi>J \\ \frac{J-I}{J-\Phi} & J \geq \Phi>I\end{array} \forall \in R\right.$

Depending on the user's expected travel time $(I)$, this model illustrates that during traveling from $s$ to $d($ where $s \neq d)$, if $I$ is higher than or equals the actual time cost $\Phi(r)$ on the router, the user will have the highest fulfillment level and the dissatisfaction function will take the value of 0 . If the user's highest expected time cost $(J)$ is smaller than the actual time cost, the user will be highly dissatisfied by traveling on this route. Thus, the fulfillment function will be zeroed.

\section{Decision model}

\subsection{Fuzzy routing optimization formation}

In the routing field, combining and weighting multiple criteria that is used to generate alternate routes is very important since the weighting process may totally change the optimality of the path. It is a multiple optimization problem with time-dependent route cost. Finding the efficient path have been widely estimated form minimizing linear combinations of different criteria. Although it is an easy weighting method to compute the optimal route, but it overemphasizes the criteria that is weighted most. In other words, the linear combination of the decision criteria tends to ignore the reduction in marginal utility since it always underlines the most weighted criterion [17].

Yager (1978) [18] introduced a fuzzy decision optimization model for the multi objective optimization with including differing degrees of importance to different objectives. i.e., involving the absence of sharply defined criteria. For instance, if the negotiation is between the travel time and travel distance only and minimizing the travel time is weighted higher than the travel distance, it is not logic to drive twice the distance to save 7 seconds of travel time that are needed to be on the optimal route even if minimizing the travel time is the major criterion. This fuzzy optimization argument situation is the classical problem of maximization of the minimums which is formed by Yager (1978)[18] as fuzzy optimization model. In the eco-routing model in this research, it can be formed as:

$D\left(r^{*}\right)=\underset{r \in R}{\operatorname{Maximize}} D(r)$

$r^{*}$ is determined by optimizing the fuzzy decision variable $D$. In which, non-negative number represents the weights criterion is defined. Each criterion is raised to this weight as a power before combining in the decision variable. These weights are determined based on the views of a group of experts in social and transportation planning field in addition to decision makers. In the routing model in this research, the weights are $\omega_{1}, \quad \omega_{2}, \quad \omega_{3}$, and $\omega_{4}$ for $E_{\text {iopt }}(r), \theta(r), H(r)$, and $F(r)$ respectively. And $D(r)$ in this case is formed as:

$D(r)=\left\{\min _{r \in R}\left\{\left(E_{\text {iopt }}(r)\right)^{\omega 1},(\theta(r))^{\omega 2},(H(r))^{\omega 3},(F(r))^{\omega 4}\right\}\right\}(21)$

The two previous formulas illustrates that the optimal route is chosen be the following sequence:

1. For each route $r$, determine which travel cost that this route $r$ minimizes its powered value.

2. Form a set of all minimum powered values that is minimized by each route.

3. The optimal solution is the route that has the maximum value from the formed set.

\subsection{Illustrative example}

Searching the eco-route between $s$ and $d$. Assume that $R=\left\{r_{1}, r_{2}, r_{3}\right\}$. The environmental cost, the user instinctive, traffic performance, and the user preference are as shown in Table 6-5. $\omega_{1}, \omega_{2}, \omega_{3}$, and $\omega_{4}$ are $3,0.3,2$, and 0.9 in order. From the table, $D(r)$ is formed as:

$D(r)=\{\underbrace{0.008}_{r 1}, \underbrace{0.09}_{r 2}, \underbrace{0.001}_{r 3}\}$

and the optimal eco-route is the route $r_{2}$.

Table 1. Results of the example for the fuzzy eco-routing decision model

\begin{tabular}{lcccccc}
\hline Route & $E_{\text {iopt }} \mid$ & $\theta(r)$ & $H(r)$ & $F\left(r\left(E_{\text {iopt }}(r),(\theta(r))\right.\right.$ \\
\hline$r_{1}$ & 0.2 & 0.8 & 0.8 & 0.7 & 0.008 & 0.94 \\
\hline$r_{2}$ & 0.6 & 0.4 & 0.3 & 0.5 & 0.216 & 0.76 \\
\hline$r_{3}$ & 0.1 & 0.9 & 0.7 & 0.6 & 0.001 & 0.99
\end{tabular}

\begin{tabular}{lcc}
\hline Route & $(H(r))^{\omega 3}$ & $(F(r))^{\omega 4}$ \\
\hline$r_{1}$ & 0.64 & 0.73 \\
\hline$r_{2}$ & 0.09 & 0.54 \\
\hline$r_{3}$ & 0.49 & 0.63 \\
\hline
\end{tabular}




\section{Conclusions}

This paper introduced an eco-routing guidance model for vehicles on urban street networks. With concern to different travel cost on the routes, the model considered 4 costs; the environmental cost, the user instinctive, traffic performance, and the user preference. This is in order to search for a social optimum routing solution. The emission cost model is formed standing of the VT-Meso emission model which considers different pollutants emission during different driving status. The effect of flow interruption caused by the signalized intersection is considered by comparing the exact calculated arriving time at the intersection with the phase status of the traffic signal at the intersection. As a mean to encourage users to follow the eco-routing advice, an incentive representing the fuel consumption discount indicator is involved in the decision variable. The traffic performance of the network is also considered. Traffic density ratio is minimized trough all allowable routes from the origin to the destination. Also, the user dissatisfaction is minimized trough considering minimizing a travel time dissatisfaction function. For optimizing the decision variable, a fuzzy decision optimization is introduced to overcome shortcomings of the linear weighting combination which tends to ignore the reduction in marginal utility since it always underlines the most weighted criterion. The fuzzy multi objective optimization is formed as maximization of the minimums of the travel costs. With involving the absence of emphasize sharply defined criteria. Finally, and illustration example is introduced to explain the application of the ecorouting optimization model.

\section{Acknowledgements}

The first author would like to acknowledge and thank E-JUST and MoHE for supporting him with scientific and financial support needed to perform this research.

\section{References}

[1] "U.S. Department of Transportation, National Intelligent Transportation System Architecture," 2007. [Online]. Available: http://www.iteris.com/itsarch/.

[2] W. Herbert and F. Mili, "Route guidance: state of the art vs. state of the practice," Intell. Veh. Symp. 2008 IEEE, 2008.
[3] R. Zhang, Z. Li, C. Feng, and S. Jiang, "Traffic Routing Guidance Algorithm Based on Backpressure with a Trade-Off between User Satisfaction and Traffic Load," Veh. Technol. Conf. (VTC Fall), 2012 IEEE, pp. 1-5, 2012.

[4] J. van Kampen, "Route Guidance and Signal Control Based on the Back-Pressure Algorithm," Delft University of Technology, 2015.

[5] K. Ahn and H. Rakha, "The effects of route choice decisions on vehicle energy consumption and emissions," Transp. Res. Part D Transp. Environ., vol. 13, no. 3, pp. 151-167, 2008.

[6] M. Barth, K. Boriboonsomsin, and A. Vu, "Environmentallyfriendly navigation," Intell. Transp. Syst. Conf. IEEE, 2007., 2007.

[7] E. Ericsson, H. Larsson, and K. Brundell-Freij, "Optimizing route choice for lowest fuel consumption-Potential effects of a new driver support tool," Transp. Res. Part C Emerg. Technol., vol. 14 , no. 6 , pp. 369-383, 2006.

[8] K. Boriboonsomsin and M. Barth, "Eco-routing navigation system based on multisource historical and real-time traffic information," Intell. Transp. Syst. IEEE Trans., vol. 13, no. 4, pp. 1694-1704, 2012.

[9] H. Rakha, K. Ahn, and K. Moran, "Integration framework for modeling eco-routing strategies: logic and preliminary results," Int. J. ..., vol. 1, no. 3, pp. 259-274, 2012.

[10]A. Chen, Z. Zhou, and S. Ryu, "Modeling physical and environmental side constraints in traffic equilibrium problem," Int. J. Sustain. Transp., vol. 5, no. 3, pp. 172-197, 2011.

[11]Y. Nie and Q. Li, "An eco-routing model considering microscopic vehicle operating conditions," Transp. Res. Part B Methodol., vol. 55, pp. 154-170, 2013.

[12]J. Sun and H. X. Liu, "Stochastic eco-routing in a signalized traffic network," Transp. Res. Part C Emerg. Technol., vol. 59, pp. 32-47, 2015.

[13]B. Son, J. H. Maeng, Y. J. Han, and B. G. Lee, "Pitfall in Using Average Travel Speed in Traffic Signalized Intersection Networks," Mob. Ad-hoc Sens. Networks, pp. 1059-1064, 2005.

[14]H. Rakha, H. Yue, and F. Dion, "VT-Meso model framework for estimating hot-stabilized light-duty vehicle fuel consumption and emission rates," Can. J. Civ. Eng., vol. 38, no. 11, pp. 1274-1286, 2011.

[15]H. Rakha, K. Ahn, and A. Trani, "Development of VT-Micro model for estimating hot stabilized light duty vehicle and truck emissions," Transp. Res. Part D Transp. Environ., vol. 9, no. 1, pp. 49-74, 2004.

[16]B. D. Greenshields, J. Bibbins, W. Channing, and H. Miller, “A study of traffic capacity," Highway Research Board Proceedings. pp. 448-477, 1935.

[17]J. Wahle, O. Annen, C. Schuster, L. Neubert, and M. Schreckenberg, "A dynamic route guidance system based on real traffic data," Eur. J. Oper. Res., vol. 131, no. 2, pp. $302-$ 308, 2001.

[18]R. R. Yager, "FUZZY DECISION MAKING INCLUDING UNEQUAL OBJECTIVES," Fuzzy Sets Syst., vol. 1, pp. 87$95,1978$. 